\title{
Archéopages
}

Archéopages

Archéologie et société

$36 \mid 01 / 2013$

Exotismes

\section{Un culte exotique en Gaule. L'exemple du temple de Mithra à Juliomagus - Angers}

Jean Brodeur

\section{(2) OpenEdition}

1 Journals

Édition électronique

URL : https://journals.openedition.org/archeopages/204

DOI : 10.4000/archeopages.204

ISSN : 2269-9872

Éditeur

INRAP - Institut national de recherches archéologiques préventives

Édition imprimée

Date de publication : 1 novembre 2013

Pagination : 10-15

ISSN : 1622-8545

\section{Référence électronique}

Jean Brodeur, « Un culte exotique en Gaule. L'exemple du temple de Mithra à Juliomagus - Angers »,

Archéopages [En ligne], 36 | 01/2013, mis en ligne le 01 janvier 2015, consulté le 21 janvier 2022. URL http://journals.openedition.org/archeopages/204 ; DOI : https://doi.org/10.4000/archeopages.204 


\title{
Un culte exotique en Gaule L'exemple du temple de Mithra à Juliomagus - Angers
}

\author{
Jean Brodeur Inrap
}

Les conquêtes territoriales de Rome ont généré la diffusion d'un mode de vie d'essence méditerranéenne qui a bouleversé l'organisation et les mœurs de la société gauloise. Le développement des voies de communication sur un territoire pacifié, sécurisé, a entraîné une augmentation des échanges commerciaux. L'arrivée de produits nouveaux, en grande quantité et à un meilleur coût, a ainsi permis à un nombre de plus en plus important d'habitants de consommer, voire d'acquérir parfois un goût très prononcé pour l'un d'entre eux. Le vin, produit « exotique», déjà très prisé par les élites gauloises qui en buvaient avec extravagance lors de banquets rituels, comme en témoignent les milliers de tessons d'amphores livrés par les sanctuaires indigènes et les textes d'auteurs grecs et latins, en est un exemple flagrant. La présence des récipients vinaires dans les premiers niveaux archéologiques urbains est d'ailleurs un bon indicateur de romanisation. Le goût pour ce breuvage est tel que l'on va très vite cultiver cette plante sauvage et créer un vignoble qui donnera naissance aux premiers crus. $\mathrm{Si}$, dans cet Empire, les biens matériels s'échangent, se déplacent, il en est de même pour les hommes, les idées et les croyances.

\section{Goût ou traces d'exotisme?}

Par goût de l'exotisme, il faut entendre ici une acception antique de la formulation, soit l'attirance particulière pour tout ce qui vient du «dehors », ce qui est «étranger» aux habitudes ou à des coutumes de vie séculaires attachées à un lieu. Le champ de perception est donc extrêmement large et ne se réduit pas à la définition d'aujourd'hui, issue de la période romantique où, en pleine expansion coloniale, il s'agissait de valoriser la suprématie de l'Occident sur l'Orient, dont certains aspects seulement, culturels ou artistiques par exemple, méritaient un intérêt, une curiosité qui allèrent parfois jusqu'à transformer ce "goût de l'exotisme» en mode.

En Gaule, pour les populations conquises, au final, tout ou presque était exotisme; reste à déterminer si les traces matérielles recueillies par l'archéologue sont le reflet d'une attirance particulière ou la satisfaction simple de nouveaux besoins facilitée par la mise en place d'un ample réseau commercial. Le monde romain a sans doute réussi habilement à réunir les deux tendances. Bien des exemples attestent ainsi d'une attirance pour l'exotisme : l'importation d'animaux exotiques vus comme «fantastiques» - certains retenus pour leur force ou leur férocité qui rendaient les jeux encore plus attractifs -, d'esclaves de contrées lointaines en raison de telle ou telle qualité, d'œuvres d'art appréciées pour leur valeur esthétique comme à Rome, à lépoque césarienne, où l'importation d'objets archaïques découverts lors de la fondation de la ville de Corinthe donne naissance à un véritable marché des «antiquités » et à une mode. Mais la nouvelle possibilité qui était donnée d'obtenir ces biens, dont la valeur ajoutée était leur provenance extérieure, consacrait le pouvoir d'un Empire qui l'avait permis.

\section{Vers de nouvelles formes de consommation}

L'hivernage de la VIIe légion, commandée par le jeune Publius Crassus en 57-56 avant notre ère dans le territoire andécave, constitua sans aucun doute une amorce et un vecteur de «nouvelles formes de consommation ». On peut imaginer la curiosité des autochtones face à cette concentration de plusieurs milliers de militaires romains, peut-être bien plus nombreux qu'eux, aux comportements, à l'allure, même, «étranges » pour la majorité de ces Gaulois. Pourtant, quelques décennies plus 


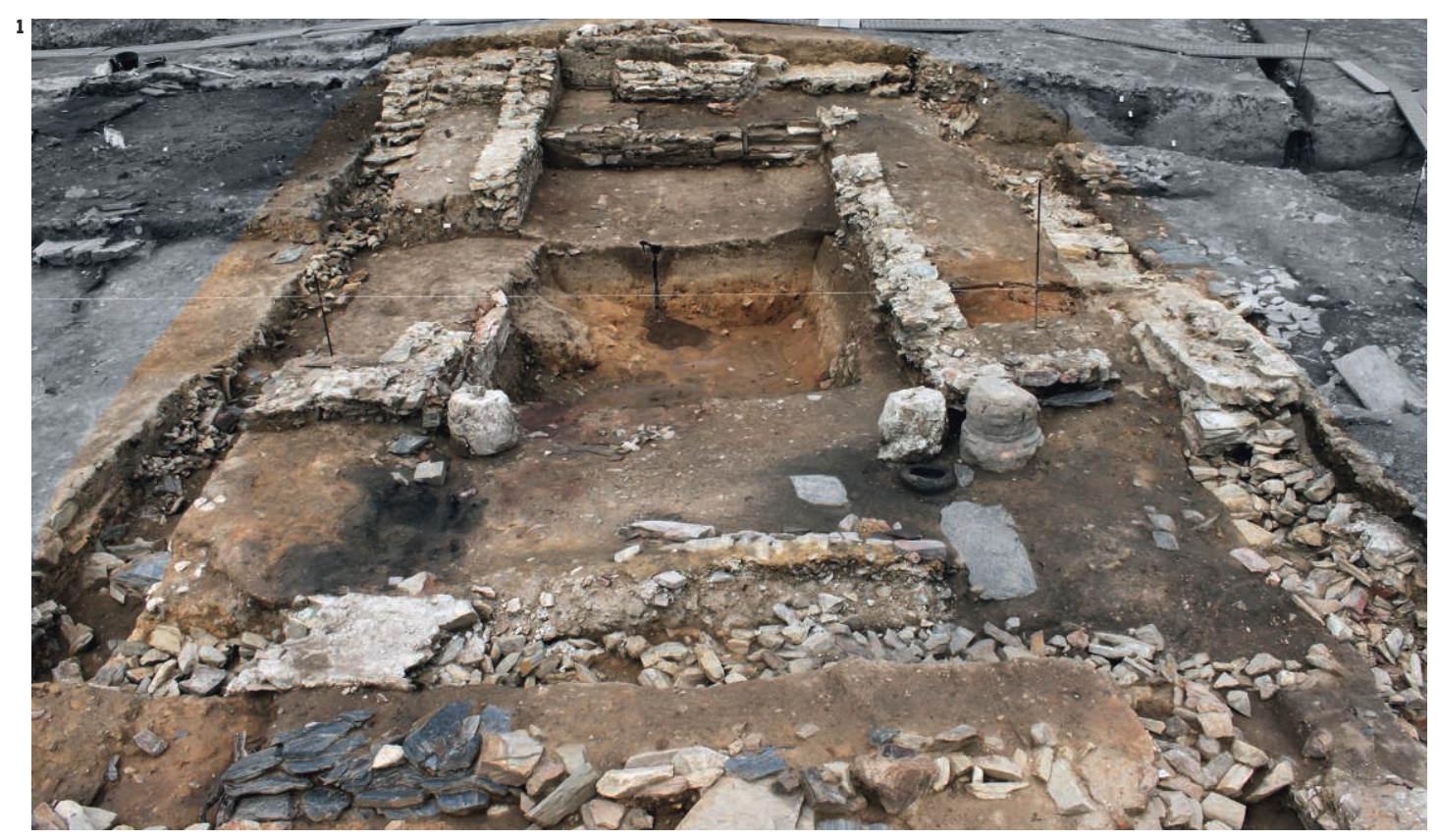

1. Vue générale

du spelaeum,

emmarchement,

base des dadophores et

d'un autel, les banquettes

et en fond le podium

supportant le socle

du bas-relief.

2. Vase avec inscription

votive: [à l'empereur et]

au Dieu invaincu Mythra

[]s Genialis, citoyen

ambien (?), en raison

d'un vœu accompli,

a fait don [ aux

(non déchiffré),

à tout, au lieu, de

(non déchiffré)

3. Plan général

du spelaeum, dernière

phase avant l'abandon

fin du Ive siècle ou

tout début du ve siècle.

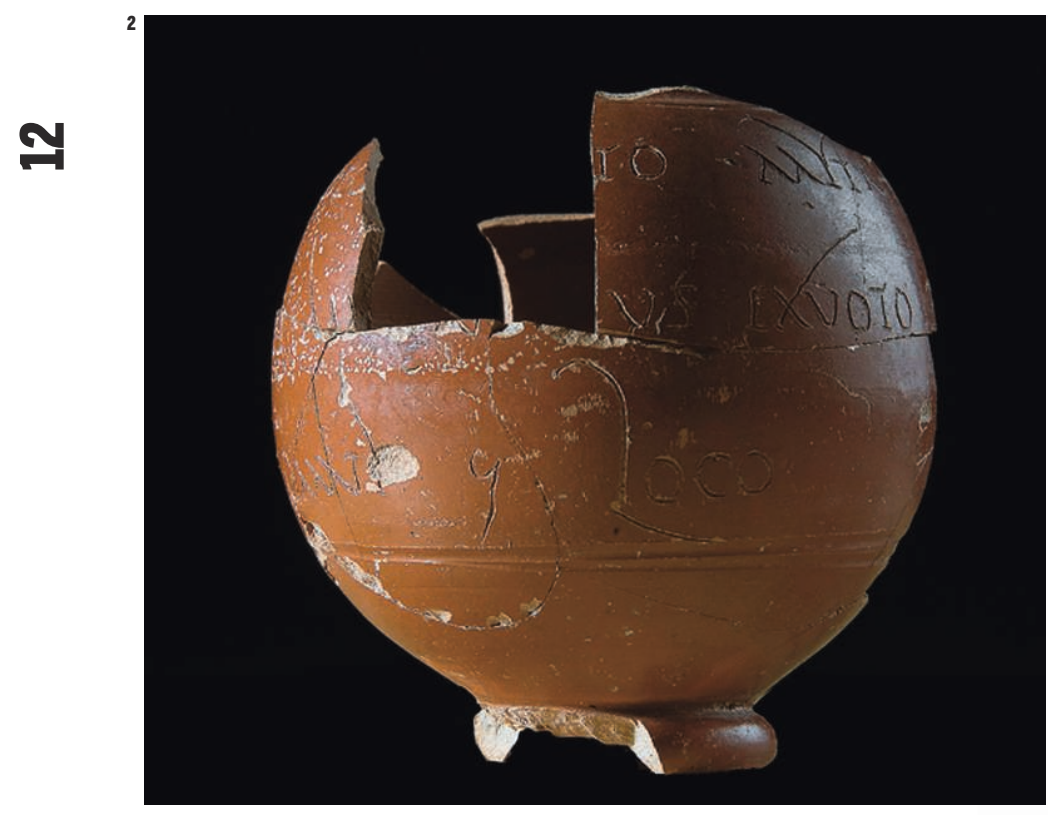

3 
pour disparaître dans la deuxième moitié, voire à la fin $\mathrm{du} \mathrm{IV}^{\mathrm{e}}$ siècle, la promulgation de l'édit d'interdiction des cultes païens jusque dans la sphère privée ${ }^{2}$ par Théodose en 392 engageant le déclin comme le suggère Saint Jérôme au début $\mathrm{du}_{\mathrm{v}}^{\mathrm{e}}$ siècle (Saint Jérôme, Lettre à Laeta, CVII, 2).

Depuis les origines, Mithra est associé au soleil, l'Helios des Grecs, puis le Sol des Latins. Helios lui donne l'ordre d'immoler le taureau, à la fois image $\mathrm{du}$ «Premier mortel» et du Mal, dans une chasse qui symbolise la lutte du Bien. Progressivement, de serviteur du dieu suprême, Mithra en devient l'égal, voire s'assimile à $S o l$, d'où la mention dans les ex-voto de «Sol invictus», le soleil invaincu. La mise à mort du taureau, la «tauroctonie», va devenir l'image principale et indissociable du mithraïsme. Elle a pour but de régénérer, de faire renaître la vie dans l'univers. Le dieu accompagné de son chien, prévenu par le corbeau, messager d'Helios, plonge son glaive au niveau de l'encolure de l'animal capturé et emmené dans une grotte. Le sang jaillissant, tout comme la semence, viennent irriguer la terre d'où surgit la vie sous la forme de plantes, d'animaux et d'eaux bienfaisantes. Un scorpion maléfique lui pince les testicules pour tenter d'empêcher cette renaissance. La scène se passe dans une grotte qui rappelle celle native du dieu. En effet, Mithra est petrogenus, c'est-à-dire né de la pierre comme le feu et donc la lumière. Dans le mithraeum, la chapelle, ou spelaeum, va reproduire cet antre sacré, d'où sa position semi, voire totalement enterrée. L'espace peut être voûté sans que ce soit toujours le cas. En revanche, il règne toujours une pénombre, voire une obscurité, qui renforce le mystère des lieux. Le luminaire est ainsi omniprésent dans le mobilier découvert [cf. encadré p.16]. La voûte est parfois ornée d'un ciel étoilé alors qu'une représentation de Mithra immolant le taureau figure toujours, souvent dans une sorte d'alcôve décorée des signes du zodiaque, à l'extrémité du spelaeum. Deux petits personnages appelés dadophores, littéralement les porteurs de torches, nommés Cautes et Cautopates, accompagnent le dieu. Cautes brandit une torche levée alors que Cautopates la dirige vers le sol pour symboliser ainsi le jour et la nuit, le lever et le coucher du soleil. Dans certaines représentations, c'est Mithra lui-même qui arbore la torche, renvoyant ainsi à l'image de l'étincelle ou première lumière de la vie. Le commandement d'Helios accompli, le dieu célèbre sa victoire par un banquet que les adeptes vont reproduire dans la liturgie. C'est manifestement le moment fort du culte. Le spelaeum se présente ainsi presque comme un triclinium, avec des banquettes latérales pour accueillir les adeptes au repas rituel, face à la tauroctonie. Les mets, parmi lesquels des animaux sacrifiés, sont préparés in situ dans une cuisine dont la localisation est variable (apparitorium, vestibule). Mithra quitte alors la terre dans le char solaire pour rejoindre le royaume des dieux.

Le culte autour du mythe s'articulait au travers de rites pratiqués par la communauté d'adeptes.
Tout dans la composition apparaît comme exotique à la culture d'alors. Mithra, Cautes et Cautopates arborent ainsi une tenue vestimentaire, en particulier le bonnet phrygien, d'origine orientale. En toute logique, et c'est le cas à Angers, certains mobiliers [cf. encadré p.18] font appel à des références tout aussi exotiques. L'architecture des mithraea est évidemment conçue afin de répondre à la liturgie et au nombre d'adeptes. Le plan rectangulaire du spelaeum, en forme de nef, avec les banquettes latérales, participent d'une mise en scène de la tauroctonie parfaitement adaptée à la célébration des banquets (Brodeur et al., 2013) [ill. 3]. Le syncrétisme avec le soleil a imposé un certain nombre de références à l'astre, aux équinoxes et aux solstices. Depuis Aurélien, celui d'hiver, par exemple, marque la naissance de Mithra le 25 décembre. L'orientation est-ouest des chapelles a été recherchée, même si elle ne paraît pas de règle, à en juger par la variabilité dans ce domaine.

L'organisation d'une communauté d'adeptes transparaît dans un texte de saint Jérôme au $\mathrm{V}^{\mathrm{e}}$ siècle (Saint Jérôme, Lettre à Laeta, CVII), confirmé par des inscriptions présentes dans certains mithraea. Parmi les grades, les figures « exotiques» sont évidemment présentes. Ainsi, sept degrés, en lien avec des planètes tutélaires et des symboles, doivent être gravis par le nouvel arrivant. Le premier est toujours celui du Corbeau, viennent ensuite le Caché (cryphius), le Soldat (miles) le Lion, le Perse, l'Héliodrome (messager du soleil) et le père (pater). D'autres sources font apparaître le Fiancé (nymphus) immédiatement après le corbeau avec, en troisième position, le miles, soldat, omis par saint Jérôme. Comme le montrent les fresques d'une caverne de Capoue, le passage d'un grade à l'autre s'accompagne de rites symboliques comme la soumission à une bastonnade (Vermaseren, 1960) ou autre épreuve physique. Selon les lieux, l'initiation peut s'effectuer dans le spelaeum ou dans un endroit annexe assimilable à une schola. L'adhésion à Mithra est par ailleurs sanctionnée par une sorte de baptême décrié par Tertullien : «Lui aussi, il baptise ceux qui croient en lui, ses fidèles : de ce bain viendra l'expiation des fautes, et, si je me souviens encore de Mithra, il marque là au front ses soldats...» (Tertullien, Traité de la prescription contre les hérétiques, XL).

Mais là encore, comme précédemment, on doit pondérer l'importance d'un goût particulier pour ces cultes exotiques. Ainsi, la communauté est peu nombreuse, restreinte à quelques dizaines d'adeptes dont d'ailleurs les femmes sont, semble-t-il, totalement exclues ${ }^{\mathbf{3}}$. Dieu suprême et solaire, qui promet une renaissance, Mithra est adopté principalement par les militaires et les marchands qui vont diffuser son culte au sein de l'Empire. Ainsi, un inventaire des inscriptions mithriaques réalisé en 2006 fait apparaître que sur 210 dédicants, 81 \% sont des militaires (Cébeillac-Gervasoni et al., 2006, p. 273). La cartographie proposée par Robert du Code théodosien, cité par
Loriot X., Badel C. (dir.),
1993.
3. Même si un doute est
permis quant à l'adhésion
à une communauté
selon une affirmation
de Tertullien, Traité de
la prescription contre les
hérétiques, XL, Musagora
(trad.) : «Il (Mithra) a
aussi ses vierges, il a aussi
ses continents ». 

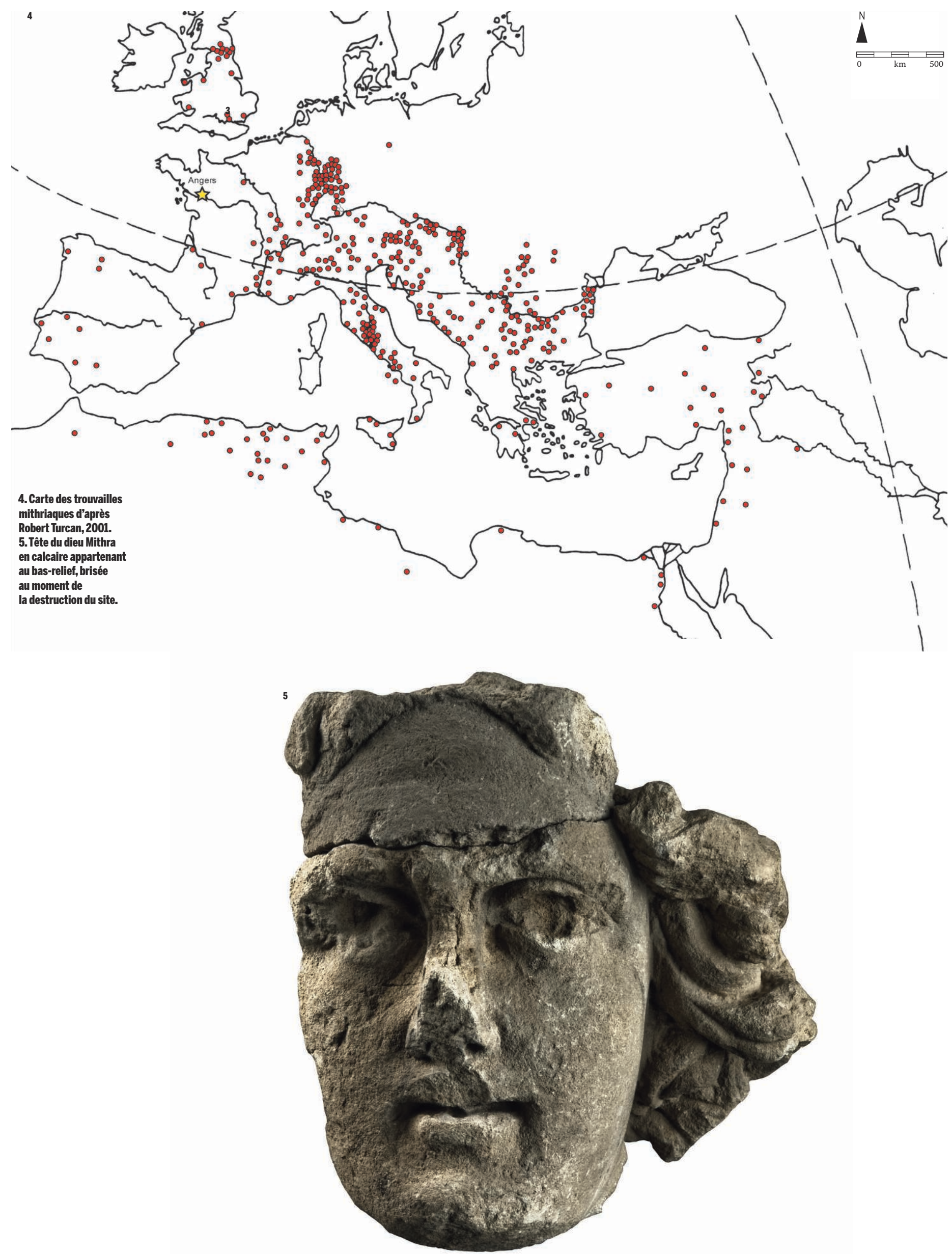
Turcan met en outre en évidence une concentration de découvertes mithriaques le long du limes ou dans les lieux où une présence militaire est reconnue. Les élites, notamment urbaines, mais pas seulement ${ }^{\mathbf{4}}$, sont toutefois séduites par cette religion initiatique qui n'est d'ailleurs pas fermée à des personnes de plus modeste condition ou statut, comme des artisans et même des esclaves. À Angers, la nature des dépôts (monnayage, armement : hache, pointes de lances et de flèches, cotte de maille ou fibules cruciformes arborés par des dignitaires de l'Empire) indique une clientèle principalement militaire dont la présence est reconnue ailleurs dans la ville (Mortreau, 2008, p. 9-39). En outre, si Mithra apparaît bien comme un dieu suprême et solaire, il ne tend nullement au monothéisme comme l'illustre parfaitement l'inscription funéraire du noble Prétextat en 384 : «Aux dieux Mânes, Vettius Agorius Praetextatus, augure, pontife de Vesta, pontife du Soleil, quindécemvir, curialis d'Hercule, consacré à Liber et aux déesses d'Eleusis, hiérophante, néocore, taurobolisé, père des pères (le plus haut degré de l'initiation mithriaque)...» (Badel, Loriot, 1993, p. 670). C'est ce qui le différencie du dieu d'une autre religion exotique auquel il va s'opposer : le christianisme, qui ne peut souffrir cette diversité.

S'il existe des similitudes dogmatiques entre mithriacisme et christianisme, contre lesquelles d'ailleurs les premiers apologistes chrétiens comme Justin et Tertullien ont rapidement réagi, les deux cultes s'opposent radicalement quant à leur ouverture sur le monde et la pensée. Une grande tolérance de la part des autorités accompagne donc cette dévotion qui ne remet pas en cause l'autorité et la divinité de l'empereur. Ainsi, en 250, sous l'empereur Dèce, l'obligation pour tout citoyen de prouver, par l'obtention d'un certificat de l'administration, la pratique d'un sacrifice aux dieux entraîne une persécution des chrétiens puisqu'elle signifie un reniement de la foi (Badel, Loriot, 1993, p. 659). La situation est particulièrement délicate pour les militaires, puisque l'on va même jusqu'à se poser la question: peut-on être soldat et chrétien? Et Tertullien de s'accorder justement avec «l'attitude d'un soldat qui lors d'une cérémonie militaire et religieuse, le donativum, sort du rang, retire sa couronne de cérémonie et confesse publiquement sa foi. Par ce geste, il choisit d'être soldat du Christ et non plus soldat de l'Empereur »
(Casaux, 2010). S'il critique tout autant

le sacramentum, véritable serment militaire lors de l'initiation du miles qui reçoit alors une couronne (Tertullien, De corona, XV, 3, 4), la seule différence est que cette action n'efface en rien l'engagement vis-à-vis de l'empereur, d'autant plus que certains César ont manifesté leur intérêt, voire une adhésion au culte mithriaque. Parmi eux, figurent de grands stratèges militaires comme Trajan, Septime Sévère, Commode ou encore Dioclétien (Freyburger et al., 2006, p. 299). Il n'est pas impossible que le rejet des militaires, certes temporaire, ait favorisé par la suite l'ancrage mithriaque de cette élite.

Il faut sans doute aussi replacer la diffusion des deux cultes dans des cadres chronologiques, en particulier en Gaule et en tenant compte de l'existence de différentes communautés chrétiennes qui forment à l'origine autant d'églises. La christianisation semble ainsi ne s'être affirmée qu'au cours du III $^{\mathrm{e}}$ siècle, « au temps de l'empereur Dèce», avec l'arrivée des premiers missionnaires venus de Rome, comme le pensait déjà Grégoire de Tours au vi ${ }^{\mathrm{e}}$ siècle (Guyon, Heijmans, 2002, p. 25). «À la différence de l'Orient, l'Italie et l'Afrique, l'Occident auraient ainsi offert au début du IV ${ }^{\mathrm{e}}$ siècle un territoire encore très largement ouvert

à la mission» (Guyon, Heijmans, 2002, p. 25). Ainsi, durant cette période, les deux cultes se pratiquent, tant que le christianisme n'aboutit pas à cette « Église universelle » (catholique en grec) véritablement formalisée avec l'édit de Théodose en 392 et l'élimination d'Eugène. À Angers, la présence de monnaies émises entre 388 et 392 jusqu'à 402, alors que le premier évêque, Defensor, est mentionné en 372, indique forcément la coexistence dans la ville du culte de Mithra et du christianisme. Comme le souligne la découverte d'Angers, qui complète la liste du très petit nombre de mithraea connus pour les Gaules (Bordeaux, Strasbourg, Biesheim, Septeuil, Mandelieu, Tirlemont (Belgique), Martigny (Suisse), etc.), l'attirance pour ce culte oriental exotique n'avait atteint que peu d'individus en raison de sa fermeture au monde, alors que son concurrent, à l'opposé ouvert et à visée universelle, en a séduit une majorité, en offrant une spiritualité salvatrice ressentie comme plus sincère qui correspondait, pourrait-on dire, au «goût» du moment. cas de Mandelieu dans les

Alpes-Maritimes, voir

Guyon J., Heijmans M.

(dir.), 2002, p. 29-30. 